\title{
Electron spectra close to a metal-to-insulator transition
}

\author{
Michał Karski ${ }^{1}$, Carsten Raas ${ }^{2}$, and Götz S. Uhrig ${ }^{2}$ \\ 1 Institut für Angewandte Physik der Universität Bonn, Wegelerstr. 8, 53115 Bonn, Germany and \\ 2 Theoretische Physik FR 7.1, Gebäude 38, Universität des Saarlandes, 66123 Saarbrücken, Germany
}

(Dated: 6 July 2005)

\begin{abstract}
A high-resolution investigation of the electron spectra close to the metal-to-insulator transition in dynamic mean-field theory is presented. An all-numerical, consistent confirmation of a smooth transition at zero temperature is provided. In particular, the separation of energy scales is verified. Unexpectedly, sharp peaks at the inner Hubbard band edges occur in the metallic regime. They are signatures of the important interaction between single-particle excitations and collective modes.
\end{abstract}

PACS numbers: 71.30.+h,71.27.+a,71.28.+d,75.40.Gb

The interplay of electronic degrees of freedom with collective modes is one of the central issues in current condensed matter physics. It is particularly intriguing when the collective modes are formed by the electrons themselves due to strong interactions. Famous examples for the complexity of such systems are the high-temperature superconductors (see, e.g., Refs. 1 and 2) and materials displaying colossal magnetoresistance (see, e.g., Refs. 3 and 4).

We focus on charge and spin degrees of freedom by considering a narrow single-band model with nearestneighbor hopping $t$ where the interaction $U$ stems from the Coulomb repulsion. For simplicity, we study the halffilled case with one electron per site. Thus the minimal model is the Hubbard model

$$
\mathcal{H}=-t \sum_{\langle i, j\rangle ; \sigma} c_{i ; \sigma}^{\dagger} c_{j ; \sigma}+U \sum_{i}\left(n_{i ; \uparrow}-1 / 2\right)\left(n_{i ; \downarrow}-1 / 2\right)
$$

where $i, j$ denote sites on a lattice with $\langle i, j\rangle$ being nearest neighbors, $\sigma \in\{\uparrow, \downarrow\}$ the spin, $c_{i ; \sigma}^{(\dagger)}$ the electron annihilation (creation), and $n_{i ; \sigma}$ their density.

Leaving aside all effects of long-range order like charge or spin density waves the system is metallic for weak interaction and insulating for strong interaction. The weakly interacting system does not have significant effects of collective modes because they are overdamped by Landau damping. The strongly interacting system is a paramagnetic insulator governed at low energies by the collective modes which are the magnetic excitations. The charge modes display a large gap of the order of $U$. Thus charge and collective modes are well separated in energy so that no significant interplay is to be expected. Hence, the regime close to the transition between the metal and the insulator is the most likely to be influenced by the interplay of single-particle and collective excitations.

An estimate shows that the two-dimensional superconducting cuprates are indeed close to the metal-insulator transition. The magnetic coupling $J$ is approximately given by $4 t^{2} / U$; empirically, one has $J \approx t / 3$ so that $U / W \approx 1.5$ where $W=8 t$ is the band width in $2 \mathrm{D}$. This can be compared to the value of about $U / W \approx 1.2$ where the paramagnetic insulating phase becomes instable due to closing of the charge gap. ${ }^{5}$
It is the aim of the present work to provide evidence that a generic paramagnetic system with values of $U / W$ in the range 1 to 1.5 constitutes a highly correlated metal with significant interplay between singleparticle and collective modes. Since a controlled treatment of finite-dimensional systems other than the onedimensional chain is not possible we study the Hubbard model on the Bethe lattice with infinite coordination number $z \rightarrow \infty$.

Scaling the hopping ${ }^{6} t=D /(2 \sqrt{z})$ leads to the dynamic mean-field theory (DMFT).$^{7,8,9}$ In DMFT the lattice problem is mapped onto an effective single impurity Anderson model (SIAM) with the self-consistency conditions that the interaction $U$, the full local Green's function $G(\omega)$ and the local self-energy $\Sigma(\omega)$ are the same in the lattice and in the SIAM. ${ }^{10}$ The DMFT as an approximation to finite-dimensional systems is by now a widely employed technique. ${ }^{11}$ It allows one to include important correlation effects in the description of real materials based on $a b$ initio density-functional theories. Hence the quantitative understanding of all features of the DMFT solution of the Hamilton operator (1) is mandatory.

At low, but finite, temperatures $T$ the phase transition between metal and insulator is of first order ${ }^{10}$ and takes place at $U_{\mathrm{c}}(T)$ (Refs. 12 and 13 ) between $U_{\mathrm{c} 1}(T)$ (instability of the insulator against infinitesimal changes in amplitude) and $U_{\mathrm{c} 2}(T)$ (instability of the metal). At $T=0$, the transition has peculiar properties. ${ }^{14,15,16,17}$ It bears features from first-order transitions: a jump in the entropy and a finite hysteresis between $U_{\mathrm{c} 1}:=U_{\mathrm{c} 1}(T=0)$ and $U_{\mathrm{c} 2}:=U_{\mathrm{c} 2}(T=0)$. But there are also secondorder features because $U_{\mathrm{c}}:=U_{\mathrm{c}}(T=0)=U_{\mathrm{c} 2}$ where the quasiparticle weight vanishes continuously and the ground-state energy $E(U)$ is differentiable. The behavior of $E(U)$ is derived from a projective DMFT, ${ }^{18}$ which is based on the hypothesis that the energetically highlying spectral features do not change at the transition from the metallic to the insulating solution (separation of energy scales).

There are many determinations of $U_{\mathrm{c} 1}$ and $U_{\mathrm{c} 2}$. They (almost) agree on $(2.39 \pm 0.02) D$ for ${ }^{19,20,21} U_{\mathrm{c} 1}$ with the outlier of $(2.225 \pm 0.025) D .{ }^{22}$ For $U_{\mathrm{c} 2}$ the values range from $2.92 D$ to $3.0 D$. $^{16,18,19,21,23,24}$ The spectral densities $\rho(\omega):=-\pi^{-1} \operatorname{Im} G(\omega)$ [density of states (DOS)] display 

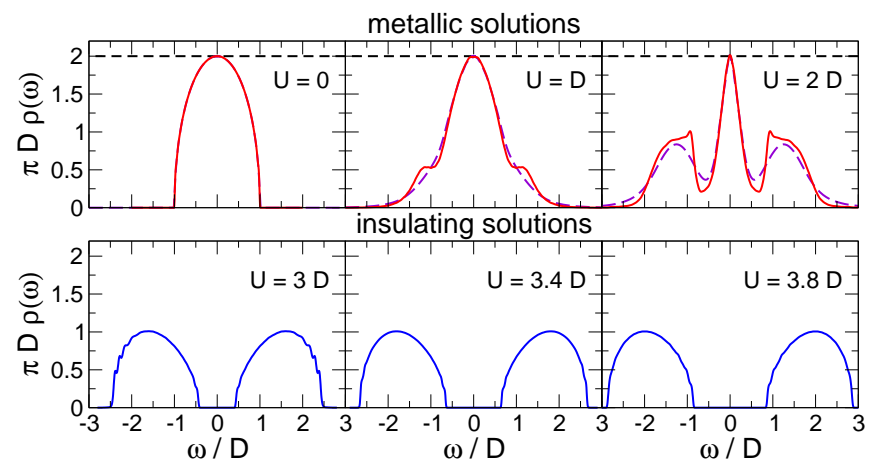

FIG. 1: (Color online) Spectral densities $\rho(\omega)$ deep in the metallic regime (upper row) and deep in the insulating regime (lower row) in DMFT for the Bethe lattice at $T=0$; dashed lines: numerical renormalization group (NRG) data (Ref. 32).

a quasiparticle peak at $\omega=0$ in the metallic solution which is pinned to its noninteracting value $\rho_{0}(0)$. But its width decreases on $U \rightarrow U_{\mathrm{c} 2} \cdot{ }^{14,16,18,21}$ The insulating solutions display the lower and the upper Hubbard bands which merge for $U \rightarrow U_{\mathrm{c} 1}$ when the single-particle gap $\Delta$ closes. ${ }^{14,19,21,22}$

Recent progress in the numerical calculation of dynamic quantities for quantum impurity models ${ }^{25,26,27,28}$ by dynamic density-matrix renormalization ${ }^{29,30,31}$ (D-DMRG) make calculations possible with wellcontrolled resolution at all energies. Thereby, spectral functions and ground-state energies become accessible which have so far eluded a quantitative determination. With the correction vector method we compute $\rho(\omega)$ broadened (convolved) by Lorentzians of width $\eta \in[0.01,0.1] D$. The unbroadened $\rho(\omega)$ is retrieved by least-bias deconvolution. ${ }^{28}$ It is used to determine the continued fraction of the bath function in the next iteration of the DMFT self-consistency cycle. ${ }^{10}$ For all $U$ about 20 iterations were performed till two subsequent $\rho(\omega)$ differed less than $\approx 10^{-3} / D$ everywhere and the ground-state energy and the double occupancy differ less than $10^{-2 \%}$. For the insulator, it is required in addition that the static gap, derived from energy differences of the finite bath representation, differs less than $1 \%$.

The D-DMRG is performed with 128 or 256 basis states. We use 120,160 , or 240 fermionic sites including the impurity in the metallic regime. For the insulating solutions we used 121 or 161 fermions. An odd number of sites implies a pole at $\omega=0$ in $\rho_{0}(\omega)$. This pole is split by the interaction. The splitting results from a pole in $\Sigma(\omega)$ at $\omega=0$. Such a solution is insulating. Hence an odd number of sites is slightly biased toward an insulator. Vice versa, an even number of sites leads to $\operatorname{Im} \Sigma(0)=0$ implying a small bias toward the metallic solution. The relative bias is estimated by the inverse number of sites: $(4-8) \times 10^{-3}$. In odd chains, we observe two almost degenerate ground-states ( $\operatorname{spin} \uparrow$ or $\downarrow$ at the interacting site) which must both be considered. Otherwise a spurious magnetic moment is generated.

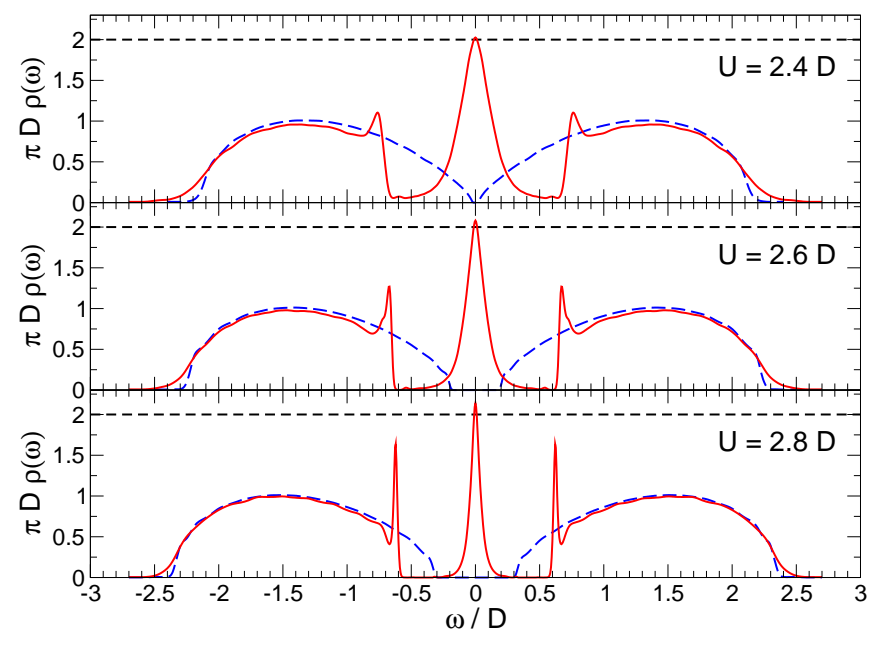

FIG. 2: (Color online) Spectral densities $\rho(\omega)$ of the metallic (solid) and the insulating (dashed) solutions between $U_{\mathrm{c} 1}$ and $U_{\mathrm{c} 2}$.

In Figs. 1 and 2, our results for metallic and insulating $\rho(\omega)$ are shown. In the metallic solutions, the narrowing of the quasiparticle band around $\omega=0$ is clearly visible. From $U \approx D$ on, the DOS displays side features which develop into the lower and upper Hubbard bands. At $U \approx 2 D$ the Hubbard bands are well separated from the quasiparticle peak at $\omega=0$ by a precursor of the gap $\Delta$ in the insulator: a pseudogap. The comparison with the NRG data from Ref. 16 shows good agreement in the quasiparticle peak but deviations in the Hubbard bands. There the DMRG data are much sharper and do not have significant tails at higher energies. This difference stems from the broadening proportional to the frequency which is inherent to the NRG algorithm. ${ }^{25,26}$

The insulating solutions display the lower and the upper Hubbard bands clearly. They agree excellently with the perturbative result ${ }^{33}$ (not shown) for $U \gtrsim 3 D$. At $U=U_{\mathrm{c} 1}=(2.38 \pm 0.02) D$ both bands touch each other. No upturn in $\rho(\omega)$ as in Ref. 22 is found when we consider the deconvolved $\rho(\omega)$ for all $\omega$. An upturn occurs only if the static gap is used. But such a procedure did not lead to stable self-consistent solutions.

In Fig. 3 the quasiparticle weight $Z$ in the metal and the single-particle gap $\Delta$ in the insulator are shown. The weight $Z=\left[1-\partial_{\omega} \operatorname{Re} \Sigma(0)\right]^{-1}$ is found from fitting the derivative of the Dyson equation $G(\omega)=G_{0}[\omega-\Sigma(\omega)]$ implying $Z^{-1}=D^{2} \partial_{\omega} G(0) / 2$ where $G_{0}(\omega)$ is the bare local Green's function of the lattice. The gap $\Delta$ is found from a fit proportional to $\sqrt{\omega-\Delta}$ to the sharp uprise of the DOS at the inner band edges. The DMRG data agree excellently with the perturbative results where the respective perturbation holds. The comparison to the NRG data shows that the broader high energy features reduce $Z$ to some extent so that the NRG weight stays below the DMRG data.

From the power-law fit $\Delta=\left(U-U_{\mathrm{c} 1}\right)^{\zeta}\left[a_{1}+a_{2}\left(U-U_{\mathrm{c} 1}\right)\right]$ shown in Fig. 3 we find $U_{\mathrm{c} 1}=(2.38 \pm 0.02) D$ in perfect 


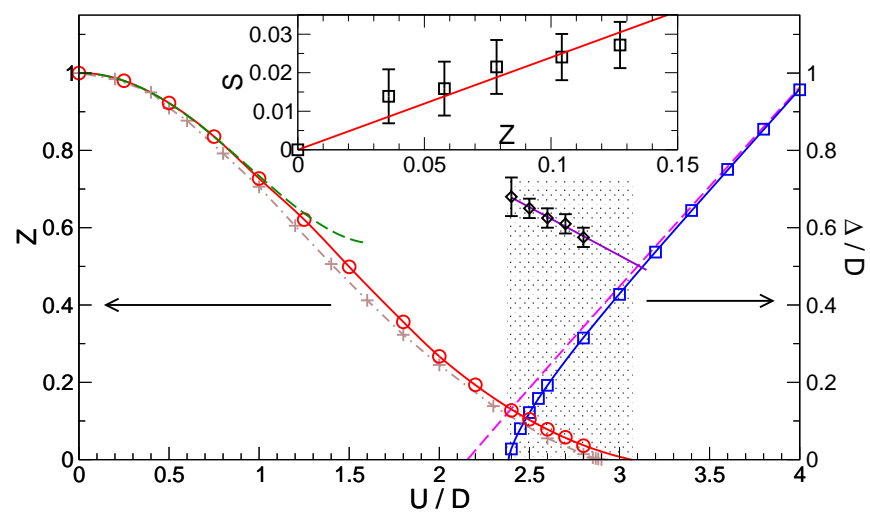

FIG. 3: (Color online) Dotted area: region of two solutions. Left curves: metallic quasiparticle weight $Z$; line with circles: interpolated DMRG, line with pluses: $\mathrm{NRG}^{16}$; dashed line: perturbation up to $U^{4}$ (Ref. 25). Right curves: insulating gap $\Delta$ or pseudogap in the metal (line with diamonds); line with squares: DMRG; dashed line: perturbation up to $1 / U^{2}$ (Ref. 33). Inset: weight $S$ of the peaks at the inner Hubbard band edges.

agreement with most of the previous results. ${ }^{19,20,21}$ The exponent is found to be $\zeta=0.72 \pm 0.05$. The value for $U_{\mathrm{c} 2}=(3.07 \pm 0.1) D$ is determined reliably from a secondorder extrapolation shown in Fig. 3. The value of $U_{\mathrm{c} 2}$ agrees well with the previous results. ${ }^{16,18,19,21,23,24} \mathrm{We}$ attribute the small deviation to the enhanced accuracy of our D-DMRG algorithm.

Figure 2 nicely shows the evolution of the metallic and the insulating $\rho(\omega)$ between $U_{\mathrm{c} 1}$ and $U_{\mathrm{c} 2}$. It represents the first all-numerical high-precision confirmation of the hypothesis of the separation of energy scales. Clearly, the metallic Hubbard bands at higher energies approach the insulating ones for $U \rightarrow U_{\mathrm{c} 2}$. Differences remain at the inner band edges and around $\omega=0$ as long as $U<U_{\mathrm{c} 2}$. The frequency of the sharp uprise in $\rho(\omega)$ at the inner edges in the metallic solutions defines the pseudogap. Its evolution (line with diamonds in Fig. 3) shows that it tends to the insulating gap at $U=U_{\mathrm{c} 2}$. This corroborates strongly that the metallic $\rho(\omega)$ approaches the insulating $\rho(\omega)$ for $U \rightarrow U_{\mathrm{c} 2}$. Remarkably, the metal and the insulator have the same single-particle correlations at $U=U_{\mathrm{c} 2}$, which is again a feature of a second-order phase transition.

From equations of motion ${ }^{22}$ one obtains the groundstate energy per site

$$
\frac{E(U)}{L}=\sum_{\sigma}\left\langle c_{i ; \sigma}^{\dagger}\left[c_{i ; \sigma}, \mathcal{H}\right]\right\rangle-U\left(\bar{d}(U)-\frac{1}{4}\right)
$$

where $\bar{d}(U)$ is the double occupancy $\left\langle n_{i ; \uparrow} n_{i ; \downarrow}\right\rangle$. All the local expectation values are computed directly at the interaction site of the SIAM at self-consistency. Then they equal the local expectation values on the lattice. In Fig. $4, E(U)$ and $\bar{d}(U)$ are depicted. The equality of $\bar{d}(U)$ in the SIAM [data points and interpolations (solid lines)] and in the lattice, obtained from $\bar{d}(U)=\partial_{U} E+1 / 4$

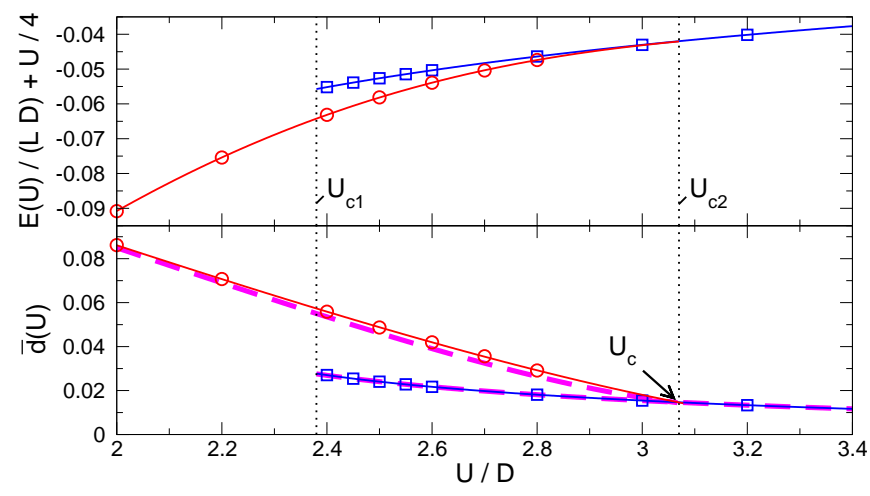

FIG. 4: (Color online) Upper panel: ground-state energy $E(U)$ in the insulator (squares) and in the metal (circles). Lower panel: corresponding double occupancy $\bar{d}(U)$. At $U_{\mathrm{c}}=$ $(3.07 \pm 0.04) D$ the double occupancies intersect. The dashed lines depict the derivative of the interpolations for $E(U)$.

(dashed lines), is a very sensitive check of the data quality. It is perfectly fulfilled in the insulator (difference $\lesssim 2 \times 10^{-4}$ ). The comparison of energies after integrating $\bar{d}(U)$ for $U \in[2.4,4.0] D$ as in Ref. 22 shows agreement within $8 \times 10^{-6} D$. In the metal, our check for $\bar{d}(U)$ works very well, but not perfectly (difference $\lesssim 3 \times 10^{-3}$ ). The remaining deviation is a finite-size effect of the bath because the deviation decreases on increasing the number of fermionic sites.

Assuming a differentiable behavior of $E(U)$ we deduce the critical interaction $U_{\mathrm{c}}=(3.07 \pm 0.04) D$ from the intersection of the double occupancies. The corresponding metallic and insulating energies differ less than $10^{-4} D$. This justifies the assumption of differentiability a posteriori. The agreement of $U_{\mathrm{c}}$ and $U_{\mathrm{c} 2}$ in our numerical treatment proves the consistency of our data. Thereby, the previously proposed scenario ${ }^{14,15,18}$ for the metalinsulator transition is numerically confirmed.

An unexpected feature in our metallic solution are the sharp peaks at the inner edges of the Hubbard bands (see Fig. 2 and Fig. 1 for $U=2 D$ ). The only previous evidence for similar features were weak shoulders in $\mathrm{NRG}^{16}$ and quantum Monte Carlo ${ }^{13}$ data. Based on the following arguments we interpret it as the signature of a collective mode.

Since the sharp peaks occur only in the metal the heavy quasiparticles must be involved. But the peaks are located at relatively high energies compared to the quasiparticle band. So a binding or antibinding phenomenon with something else must take place. Since the peaks are very sharp (to numerical accuracy for $U \gtrsim 2.6 D$ ) we conclude that an immobile, local mode is involved. Spin excitations like spin waves are the best-known collective excitations in Hubbard systems. In infinite dimensions, they are indeed dispersionless. ${ }^{34}$ In absence of any magnetic order they are located at zero energy in the insulator because the Hartree term vanishes. ${ }^{7}$ By continuity, we deduce that their energy is very low also in the metal with an estimated upper bound of $0.2 D$ deduced from the 
energy of a spin wave in the long-range ordered Néel state which is $z J / 2 \approx D^{2} /(2 U) \approx 0.2 D$ at $U=2.5 D$. Hence, we conclude from the energy of the sharp features that it signals the antibound state or resonance of a heavy quasiparticle with a collective spin excitation. We refer to this state as the antipolaron.

In the inset of Fig. 3 we plot the weight $S$ of the antipolaron peak as a function of $Z$. The error bars result from the numerical difficulty to resolve this sharp feature and from the analytical difficulty to separate it from the background of the Hubbard bands. For the separation, we fitted an approximate square root onset starting at the pseudogap, multiplied with a quadratic polynomial, to the Hubbard bands. The excess weight $S$ is attributed to the antipolaron peak. From the inset in Fig. 3 we conclude that $S$ vanishes linearly with $Z$, rather than quadratically or cubically. Thus the antipolaron peak vanishes proportional to the matrix element of a single quasiparticle. If $S$ had vanished quadratically (cubically) one would have concluded that two (three) quasiparticles were involved. The linear dependence of $S$ on $Z$ corroborates the scenario of an antibound state formed from one quasiparticle and one collective mode. Surely, further investigations are called for.

We point out that the antibinding between the heavy quasiparticles and the collective magnetic modes suggests an interesting answer to the so far open question why the metal forms Hubbard bands and eventually becomes in- sulating. The weight close to the Fermi level is pushed away to higher energies by a strong repulsive interaction between the low-lying quasiparticles and magnetic modes. Note that this scenario is also applicable in finite dimensions if the finite dispersion of the collective modes is accounted for.

In summary, we provided the all-numerical confirmation of the metal-insulator transition proposed earlier $^{14,15,18}$ on the basis of the hypothesis of the separation of energy scales. We found that the spectral density of the metal approaches the one of the insulator for $U \rightarrow U_{\mathrm{c} 2}$. The critical interactions $U_{\mathrm{c} 1}=(2.38 \pm 0.02) D$ and $U_{\mathrm{c} 2}=(3.07 \pm 0.1) D$ were found; the latter value coincides with $U_{\mathrm{c}}=(3.07 \pm 0.04) D$ where the ground-state energies $E(U)$ intersect differentiably. Hence, a consistent picture of a differentiable $E(U)$ emerged.

The unprecedented resolution of the spectral densities enabled us to detect sharp peaks at the inner Hubbard band edges. We interpreted them as antibound states or resonances formed from a heavy quasiparticle and a collective magnetic mode. The occurrence of such signatures in electron spectra opens up an interesting route to investigate the interplay of single-particle and collective excitations in photoelectron spectroscopy.

We thank R. Bulla for the NRG data and E. MüllerHartmann, A. Rosch, and L. H. Tjeng for helpful discussions and the DFG for financial support in SFB 608.
1 M. R. Norman and C. Pépin, Rep. Prog. Phys. 66, 1547 (2003).

2 E. Demler, W. Hanke, and S.-C. Zhang, Rev. Mod. Phys. 76, 909 (2004).

3 M. Imada, A. Fujimori, and Y. Tokura, Rev. Mod. Phys. 70, 1039 (1998).

${ }^{4}$ Y. Tokura and N. Nagaosa, Science 288, 462 (2000).

5 A. Reischl, E. Müller-Hartmann, and G. S. Uhrig, Phys. Rev. B 70, 245124 (2004).

6 W. Metzner and D. Vollhardt, Phys. Rev. Lett. 62, 324 (1989).

7 E. Müller-Hartmann, Z. Phys. B 74, 507 (1989).

8 M. Jarrell, Phys. Rev. Lett. 69, 168 (1992).

9 A. Georges and G. Kotliar, Phys. Rev. B 45, 6479 (1992).

10 A. Georges, G. Kotliar, W. Krauth, and M. J. Rozenberg, Rev. Mod. Phys. 68, 13 (1996).

11 G. Kotliar and D. Vollhardt, Physics Today 57(3), 53 (2004).

12 N.-H. Tong, S.-Q. Shen, and F.-C. Pu, Phys. Rev. B 64, 235109 (2002).

13 N. Blümer, (Ph.D. thesis, Universität Augsburg, 2002).

14 X. Y. Zhang, M. J. Rozenberg, and G. Kotliar, Phys. Rev. Lett. 70, 1666 (1993).

15 G. Kotliar, Eur. Phys. J. B 11, 27 (1999).

16 R. Bulla, Phys. Rev. Lett. 83, 136 (1999).

17 M. Potthoff, Eur. Phys. J. B 36, 335 (2003).

18 G. Moeller, Q. Si, G. Kotliar, M. Rozenberg, and D. S. Fisher, Phys. Rev. Lett. 74, 2082 (1995).
19 R. Bulla, T. A. Costi, and D. Vollhardt, Phys. Rev. B 64, 045103 (2001).

20 N. Blümer and E. Kalinowski, Phys. Rev. B 71, 195102 (2005).

21 D. J. Garcia, K. Hallberg, and M. J. Rozenberg, Phys. Rev. Lett. 93, 246403 (2004).

22 S. Nishimoto, F. Gebhard, and E. Jeckelmann, J. Phys.: Condens. Matter 16, 7063 (2004).

23 R. Bulla and M. Potthoff, Eur. Phys. J. B 13, 257 (2000).

24 N. Blümer and E. Kalinowski, Physica B 359, 648 (2005).

25 F. Gebhard, E. Jeckelmann, S. Mahlert, S. Nishimoto, and R. M. Noack, Eur. Phys. J. B 36, 491 (2003).

26 C. Raas, G. S. Uhrig, and F. B. Anders, Phys. Rev. B 69, 041102(R) (2004).

27 S. Nishimoto and E. Jeckelmann, J. Phys.: Condens. Matter 16, 613 (2004).

28 C. Raas and G. S. Uhrig, Eur. Phys. J. B 45, 293 (2005).

29 K. A. Hallberg, Phys. Rev. B 52, R9827 (1995).

30 S. Ramasesha, S. K. Pati, H. R. Krishnamurthy, Z. Shuai, and J. L. Brédas, Synthetic Metals 85, 1019 (1997).

31 T. D. Kühner and S. R. White, Phys. Rev. B 60, 335 (1999).

32 R. Bulla, private communication (2004).

33 M. P. Eastwood, F. Gebhard, E. Kalinowski, S. Nishimoto, and R. M. Noack, Eur. Phys. J. B 35, 155 (2003).

34 B. Kleine, G. S. Uhrig, and E. Müller-Hartmann, Europhys. Lett. 31, 37 (1995). 